\title{
"Neurocircuits to Behavior: The New Revolution"
}

\author{
Kerry J. Ressler, MD, PhD
}

\section{Introduction - The centrality of neural circuits to behavior}

Centuries after the idea that brain regional function was important, among the most transformative concepts in Psychiatry over the last half century is the awareness that distinct neural circuits subserve distinct behavioral functions. In fact, the idea that dynamic activity in distinct circuits underlies specific behaviors is actually fundamental and central to our current models of psychiatric functioning, and the tools to address these circuits have changed rapidly.

Progress since the 1980's in our ability to use the human neuroimaging tools of computed tomography and magnetic resonance imaging to transform understanding of the living human brain is unsurpassed in the history of the neuroscience. The interaction of individual brain regions in a dynamic fashion is now being understood using complex mathematical approaches to examine co-activation with dynamic fMRI. Many of these tools were at an early level of development 25 years ago at the initiation of the Harvard Review of Psychiatry (Andreasen, 1988; Rauch \& Renshaw, 1995), and are now fully routine in a large area of psychiatric neuroscience.

Until the $21^{\text {st }}$ century, however, the tools available for mapping and manipulating circuits in neuroscience model systems, and thus for understanding mechanisms, had been fairly consistent for most of the $20^{\text {th }}$ century. The classic approaches to determine causality were lesion studies, e.g. lesioning of the hippocampus in rodents and nonhuman primates established the primacy of this region in declarative memory formation. Other approaches included electrophysiological activation of brain regions and the use of a variety of plant lectins that allowed the visualization of specific axonal paths from one brain region to the next (e.g., Paylay and Chan-Paylay, 1976; Ralston, 1990). This technique depended on the observation that these lectins bound to membrane proteins allowing visualization with antibodies to illuminate the entire axonal and dendritic arbors of individual neurons. Despite these methods over the last decades, understanding the causal functioning of specific circuits lacked a number of critical tools: 1) the ability to identify or control specific, geneticallyidentified, types of cells; 2) the ability to activate or inhibit certain cells or cell pathways in a temporally precise fashion; 3 ) the ability to control specific cell types or cell pathways with systemic drug manipulation; and 4) the ability to combine genetic tools with cell-type and circuit-based tools. A full understanding of how specific circuits create specific behaviors

Corresponding Author: Kerry Ressler, MD, PhD, Mclean Hospital, Oaks Building 104b, Mailstop 212, 115 Mill Street, Belmont, MA 02478-1064, TEL: 617.855.4210 FAX: 617.977.4213, kressler@ mclean.harvard.edu.

Commentary for Harvard Review of Psychiatry, $25^{\text {th }}$ Anniversary January 8, 2017 
would require new tools to answer these questions. A number of such breakthroughs in the last decade have nothing less than transformed the neuroscience of behavior.

\section{Molecular Biology Transformation of Neural Circuit Methodology}

A number of different innovations have led to the current transformative toolbox available to behavioral neuroscientists. However, the first innovation was the molecular biology revolution, which has led to a large number of molecular genetic tools - beginning with transgenic mice in which specific cell populations express different genes that provide a wide range of abilities to delete or overexpress gene targets of interest.

Genetically modified viral vectors have more recently broadened the ability to express genes of interest (e.g., Heldt \& Ressler 2009). Combined with the region specificity of viral infusions, along with intersectional approaches that combine viruses with transgenic animals, even more powerful tools are available to target anterograde, retrograde, and a variety of cell-types. Commonly used genetically modified viruses include AdenoAssociated Virus, Lentivirus, and Herpes Simplex Virus approaches - they can all be easily modified with modern molecular engineering techniques, allowing a now huge range of inducible manipulations - cell type specific approaches, gene over-expression, mutant gene expression, inducible deletion, expressing antisense and other noncoding RNAs, and epigenetic regulation, in addition to intersectional trans-synaptic approaches.

The newest and possibly most transformative tools in recent years are optogenetic and chemogenetic approaches to actively manipulate targeted neurons. This toolbox now allows a mechanistic approach to determining temporally-specific, cell-type specific, circuitspecific neural regulation of behaviors. Thus, researchers are beginning to dissect the neuronal basis of behavior, at the levels of epigenetics, genetics, neural circuits, and dynamic behavioral regulation. These basic science principles of mammalian behavior are needed to translate mechanism-based understanding of behaviors to novel biology-based interventions.

\section{Optogenetic approaches}

Optogenetics - the control of neuronal firing through genetically controlled, molecularly engineered light-sensitive ion channels - has truly transformed neuroscience within the past decade. While several are credited with its discovery and initial implementation, Karl Deisseroth, a Psychiatrist Physician-Scientist from Stanford, is widely regarded as being most central to the innovation and dissemination of these techniques for dissecting neural circuits related to behavior (Boyden et al., 2005; Deisseroth, 2015).

Years earlier, genes encoding several different species of 'opsin' (light sensitive) receptors had been identified in algae and other simple organisms. It was realized that some of these receptors could be expressed in mammalian neurons with no adverse consequences, but when certain wavelengths of light (using fiber optic technology) were delivered to these neurons, they would fire action potentials. The initial versions of these 'excitatory' optogenetic channels were members of the 'Channel Rhodopsin' family. In parallel, a set of inhibitory channels were identified, initially of the 'halo-rhodopsin' family. Since the initial 
discovery, many additional protein channels have been identified, leading to a large array of specific tools for controlling neuronal activity in precise ways.

Deep brain stimulation has been a very powerful innovation for a number of refractory neuropsychiatric syndromes including depression. However Deep brain stimulation (DBS), while much more targeted regionally to circuits than ECT and transcranial magnetic stimulation, is still quite crude from the perspective of what we now know about microcircuits within neural regions. One imagines the possibility of a future psychiatric toolbox of cell-type specific, optogenetic regulated driving of specific behavioral microcircuits.

\section{Chemogenetic approaches}

A complementary approach to optogenetics, also advanced within the last decade, is the concept of chemogenetics, with the most robust model being DREADD technology (Designer Receptors Exclusively Activated by Designer Drugs) (Nawaratne et al., 2008; Roth, 2016). Instead of using light energy to directly activate ion channels, chemogenetic approaches use genetically modified G-protein-coupled receptors activated by an otherwise inert drug. This drug can then be delivered systemically or directly to the brain region of interest. Compared to optogenetics, chemogenetics have the distinct properties of: 1) being activated via a systemic drug not needing indwelling fiberoptics into the brain, and 2) activating cells in a more naturalistic, modulatory fashion with second messenger pathways as opposed to directly stimulating them or inhibiting them. Thus, while optogenetic approaches may provide a better understanding of the biophysics and direct connectivity of different neuronal systems, chemogenetic approaches may provide a better tool for understanding neurotransmitter-based modulation.

\section{Why does this matter? Implications for the future of Psychiatry}

These advances would have looked like science fiction at the time of the founding of $H R P$ just 25 years ago. While many of our diagnoses, psychotherapies, and even medications used for Psychiatric disorders are similar to what was available in the early 1990s, the way that modern neuroscientists approach dissecting the neural circuits underlying the mammalian brain and behavior have truly been transformed. That we have tools to routinely switch 'on' and 'off' specific neurons within specific microcircuits of specific brain areas with a light pulse or an otherwise inert, targeted drug remains astounding.

Appreciating the biology of bacteria and viruses was needed before modern control over infectious diseases. An understanding of basic biology of cell division and growth regulation was required for the modern evolution in cancer biology and mechanism-based targets in cancer treatment. Similarly, an understanding of the neural mechanisms (at the circuit, cell, and gene expression levels) is required before we can derive novel and powerful therapeutics targeted at the cellular basis of specific behaviors.

This revolution in psychiatric neuroscience is already changing our understanding of and approach to behavioral neuroscience and to disorders related to neural circuitry, e.g. psychiatric disease. It is difficult to know how thoroughly these approaches will alter the 
landscape of psychiatry, but it will assuredly be profound. At the molecular and cellular level, new approaches are actively being pursued that will allow receptor-based target identification, verification, and validation of new compounds that are identified not first by whether they affect a peculiar rodent behavior that may or may not have relevance to the human condition, but instead are based on shared neural circuitry and genetic commonality.

Furthermore, as implied above, the neurotherapeutics revolution may allow an extreme possibility of precision medicine. For example, current molecular genetic technology could lead to a single surgery for a one-time molecularly engineered virus manipulation of celltype specific microcircuits that are dysregulated in one individual's brain, followed by that person being able to take a pill in the future to directly 'switch on,' 'turn off,' or otherwise modulate that precise microcircuit - directly changing their perception of aversive, anhedonic, habitual or other dysregulated processes. This is truly the stuff of science fiction.

\section{Conclusions}

The neurocircuitry therapeutics revolution is rapidly advancing. The tools, from molecular engineered, cell-type specific viruses, to optogenetics and chemogenetics, continue to evolve rapidly and to transform the field of behavioral and functional neuroscience. The field of Psychiatry will need to equally rapidly develop an ethics of how to use these approaches. Furthermore, we must develop new theories and broader perspectives to understand how circuit dynamics underlying behavior fit within our current understanding of monaminergic modulation of depression and psychosis, for example. Even more, how do these perspectives complement our psychodynamic and behavioral psychotherapies and models of human behavior? Regardless of one's perspective, there is no doubt that a revolution is underway in the neuroscience of psychiatry. Our field, our therapeutics, and our ability to treat disease will most certainly appear drastically different in another 25 years from now.

\section{References}

Andreasen NC. Brain imaging: applications in psychiatry. Science. 1988; 239(4846):1381-1388. [PubMed: 3279509]

Boyden ES, Zhang F, Bamberg E, Nagel G, Deisseroth K. Millisecond-timescale,genetically targeted optical control of neural activity. Nat Neurosci. 2005; 8(9):1263-1268. [PubMed: 16116447]

Deisseroth K. Optogenetics: 10 years of microbial opsins in neuroscience. Nat Neurosci. 2015; 18(9): 1213-1225. [PubMed: 26308982]

Heldt SA, Ressler KJ. The Use of Lentiviral Vectors and Cre/loxP to Investigate the Function of Genes in Complex Behaviors. Front Mol Neurosci. 2009; 2:22. [PubMed: 20011219]

Nawaratne V, et al. New insights into the function of M4 muscarinic acetylcholine receptors gained using a novel allosteric modulator and a DREADD (designer receptor exclusively activated by a designer drug). Mol Pharmacol. 2008; 74(4):1119-1131. [PubMed: 18628403]

Palay SL, Chan-Palay V. A guide to the synaptic analysis of the neuropil. Cold Spring Harb Symp Quant Biol. 1976; 40:1-16. [PubMed: 59655]

Rauch SL, Renshaw PF. Clinical neuroimaging in psychiatry. Harv Rev Psychiatry. 1995 Mar-Apr; 2(6):297-312. 1995. [PubMed: 9384916]

Ralston HJ 3rd. Analysis of neuronal networks: a review of techniques for labeling axonal projections. J Electron Microsc Tech. 1990; 15(4):322-321. [PubMed: 1697336]

Roth BL. DREADDs for Neuroscientists. Neuron. 2016; 89(4):683-694. [PubMed: 26889809]

Harv Rev Psychiatry. Author manuscript; available in PMC 2018 March 01. 